\title{
The Gould's Belt Distances Survey
}

\author{
Laurent Loinard \\ Centro de Radioastronomía y Astrofísica, Universidad Nacional Autónoma de México, \\ Apartado Postal 3-72 (Xangari), 58089 Morelia, Michoacán, Mexico \\ email: 1.loinard@crya.unam.mx
}

\begin{abstract}
Very Long Baseline Interferometry (VLBI) observations can provide the positions of compact radio sources with an accuracy of order 50 micro-arcseconds. This is sufficient to measure the trigonometric parallax and proper motions of any object within $500 \mathrm{pc}$ of the Sun to better than a few percent. Because they are magnetically active, young stars are often associated with compact radio emission detectable using VLBI techniques. Here we show how VLBI observations have already constrained the distance to the most often studied nearby regions of star formation (Taurus, Ophiuchus, Orion, etc.) and have started to provide information about their internal structure and kinematics. We will then briefly describe a large project, the 'Gould's Belt Distances Survey,' which has been designed to provide a detailed view of star formation in the solar neighborhood using VLBI observations.
\end{abstract}

Keywords. astrometry, stars: distances, stars: formation, Galaxy: structure, solar neighborhood, radio continuum: stars

\section{Introduction}

Gould's Belt (for a recent review, see Poppel 1997) is a prominent local Galactic structure that contains most nearby $\mathrm{O}$ and $\mathrm{B}$ stars, as well as approximately 2 million solar masses of interstellar material. It is a flattened system of radius $\sim 1 \mathrm{kpc}$, inclined by roughly $18^{\circ}$ relative to the Galactic plane, and centered at approximately $100 \mathrm{pc}$ from the Sun in the direction $\ell \sim 180^{\circ}$. It is named after the American astronomer Benjamin Gould (also notable for being the first American to receive a Ph.D. in astronomy, and the founder of The Astronomical Journal), who described it in 1879. It is worth pointing out, however, that Sir John Herschel had already identified many of its salient features in the late 1840s. The stars in Gould's Belt exhibit peculiar kinematics, clearly indicative of non-uniform expansion. Its age is estimated to be of order $30 \mathrm{Myr}$, but its origin remains very unclear. An impulsive event, such as the impact of a dark matter halo cloud similar to Smith's cloud (see Lockman et al. 2008) with a large molecular cloud in the Galactic disk is a plausible suggestion. The complex kinematics of the structure, however, suggests that local events within the structure (e.g., supernova explosions) also contribute to its overall morphology.

Gould's Belt contains essentially all sites of ongoing star formation within 500 pc: Ophiuchus, Lupus, Taurus, Orion, the Aquila Rift and Serpens region, etc. Detailed studies of these regions have played, and continue to play, a central role in the development of our understanding of star formation in general. This can be seen, for instance, from the fact that in the last few years alone, several large space programs like the Spitzer C2D survey (Evans et al. 2009), the XMM Newton Extended Survey of Taurus (Güdel et al. 2008), or the Herschel Gould Belt Survey (André et al. 2010) have focused on these regions. Ancillary ground-based observations such as the JCMT Gould's Belt Survey (Ward-Thompson et al. 2007) have also been obtained. The analysis of this wealth of information has been somewhat affected by the lack of accurate distances to regions 
within Gould's Belt, which are rarely known to better than $30 \%$ and often significantly worse. This problem is compounded by the fact that several of these regions (as we shall see, the Aquila/Serpens region is a case in point) might contain structure at different distances along the line of sight.

The Gould's Belt Distances Survey is a large project aimed at measuring the astrometric elements of approximately 200 low-mass, young stars distributed across five of the most prominent star-forming regions in Gould's Belt (Ophiuchus, Taurus, Perseus, Aquila/Serpens, and Orion) using Very Long Baseline Interferometry (VLBI) multi-epoch radio observations. The immediate goal of these observations is to accurately measure the distances, three-dimensional structure, and internal kinematics of these regions. This will have important implications both for the study of star formation in general, and for the analysis of the origin of Gould's Belt. In this contribution, we will first present the technique used and a handful of results obtained towards nearby star-forming regions. We then proceed to describe the Gould's Belt Distances Survey itself, and the current status of the project.

\section{VLBI astrometry of low-mass, young stars}

VLBI (e.g., Thompson et al. 2001) is an observing technique that can readily provide astrometric accuracies of order 30-60 $\mu \mathrm{as}$, for compact radio sources of sufficient brightness $\left(T_{\mathrm{b}} \approx 10^{7} \mathrm{~K}\right)$. Such sources exist in all star-forming regions, because lowmass, young stars are often magnetically active. The gyration of relativistic electrons in the strong magnetic fields surrounding low-mass, young stars produces radio emission detectable with VLBI instruments (Dulk 1985). This emission is normally confined to regions extending only a few stellar radii around the stars, and therefore remains very compact even in the nearest star-forming regions (at distances $d \approx 100 \mathrm{pc}$ ). In high-mass star-forming regions, water and methanol masers (which are also detectable with VLBI instruments) are common and offer an interesting alternative to magnetically active, lowmass stars. Here, however, we are primarily concerned with low- and intermediate-mass star-forming regions where masers are of limited use, because they are rare and often strongly variable.

The implication of the astrometric accuracy reached in VLBI experiments for distance measurements is shown in Fig. 1, where we consider the relative parallax error $(\delta \pi / \pi)$ as a function of distance for astrometric accuracies typical of VLBI observations (30, 60, and $120 \mu \mathrm{as})$. For reference, the mean accuracy of Hipparcos (1 mas) is also shown. It can be seen that for any source within approximately $1 \mathrm{kpc}$, VLBI observations can yield accuracies of a few percent in the distance measurement. To demonstrate that this can be achieved in practice, we show in Fig. 2 the trajectory described on the plane of the sky by $\mathrm{T}$ Tau Sb, one of the southern companions of the famous object T Tauri, as characterized by multi-epoch VLBI observations (for details, see Loinard et al. 2007). That trajectory is the combination of an elliptical parallax component and the proper motion of the source, oriented towards the southeast in this specific case. Note that the entire size of the figure is only $\sim 10$ mas. The distance obtained from these observations $(146.7 \pm 0.6 \mathrm{pc})$, has an uncertainty of $0.4 \%$.

We now proceed to describe the results that have been obtained so far for nearby star-forming regions using multi-epoch VLBI observations.

\subsection{Ophiuchus}

During many years, Ophiuchus was thought to be at 165 pc (Chini 1981). More recently, however, shorter distances have been preferred. For example, de Geus et al. (1989) found 


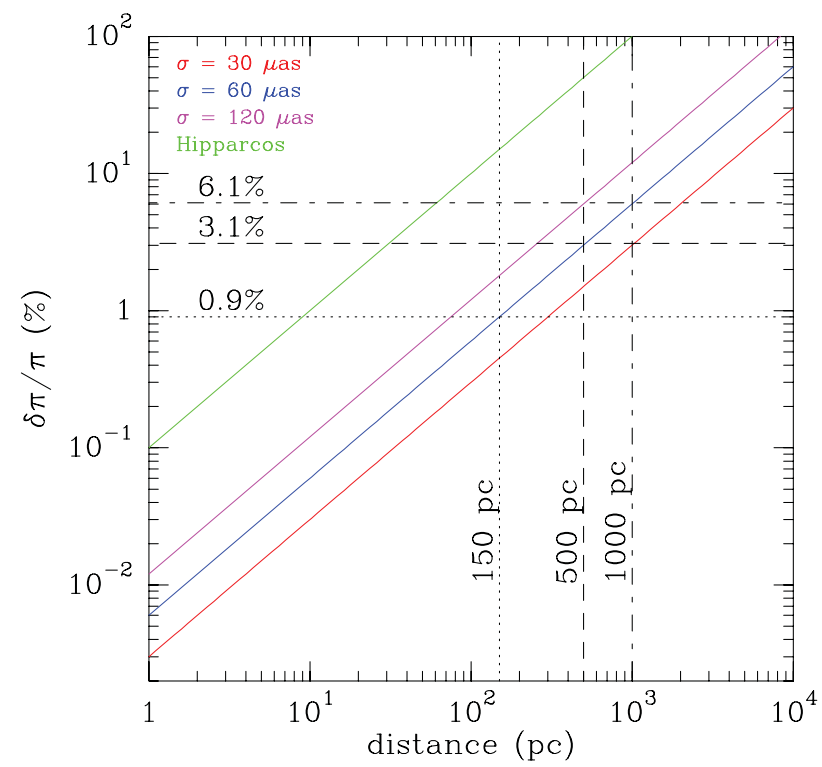

Figure 1. Relative parallax uncertainty as a function of distance for typical VLBI astrometric accuracies $(30,60,120 \mu$ as). For reference, the mean Hipparcos uncertainty (1 mas) is also shown. Representative distances $(150,500$, and 1000 pc) are indicated.

a mean photometric distance of $125 \pm 25 \mathrm{pc}$ for the stellar population associated with Ophiuchus. Knude \& Hög (1998), who examined the reddening of stars in the direction of Ophiuchus as a function of their Hipparcos distances, found a clear extinction jump at $120 \mathrm{pc}$ and argued that this corresponds to the distance of the main dust component in Ophiuchus. Using a similar method, Lombardi et al. (2008) also reported a distance of approximately $120 \mathrm{pc}$ for the Ophiuchus core.

Using VLBI observations, Loinard et al. (2008) measured the trigonometric parallax of two stars in the Ophiuchus core (S1 and DoAr21) and found a mean value of $120.0 \pm 4.5$ pc. The Ophiuchus core is only some 2 degrees across, corresponding to a linear radius of $\sim 2$ pc. The depth of the region is expected to be similar, so systematic errors in the distance to individual objects in the Ophiuchus core owing to its depth are expected to be of that order. To account for that effect, we add in quadrature $2 \mathrm{pc}$ to the formal errors reported by Loinard et al. (2008) and conclude that the distance to the Ophiuchus core should be assumed to be $120 \pm 5$ pc.

In addition to its core, Ophiuchus contains several filamentary structures known as streamers (see Wikling et al. 2008). The streamers correspond to prominent dust clouds (such as Lynds 1689, 1709, 1712, or 1729) and extend to approximately 10 pc from the core. They are thought to be physically associated with the Ophiuchus core, and should therefore be at similar distances (within approximately $10 \mathrm{pc}$, i.e. their physical extent on the plane of the sky). There are several indications that this might indeed be the case. For instance, Lombardi et al. (2008) found some indications that the streamers might be 5 to 10 pc nearer than the core. Similarly, Schaefer et al. (2008) found a distance (based on orbital modeling) to the binary young stellar object Haro 1-14c, located in the north-eastern streamer, of $111_{-18}^{+19} \mathrm{pc}$.

A somewhat puzzling result in that respect comes from the determination by Imai et al. (2007) of the trigonometric parallax of the very young stellar system IRAS 162932422 in the eastern streamer Lynds 1689. Based on VLBI observations of water masers 


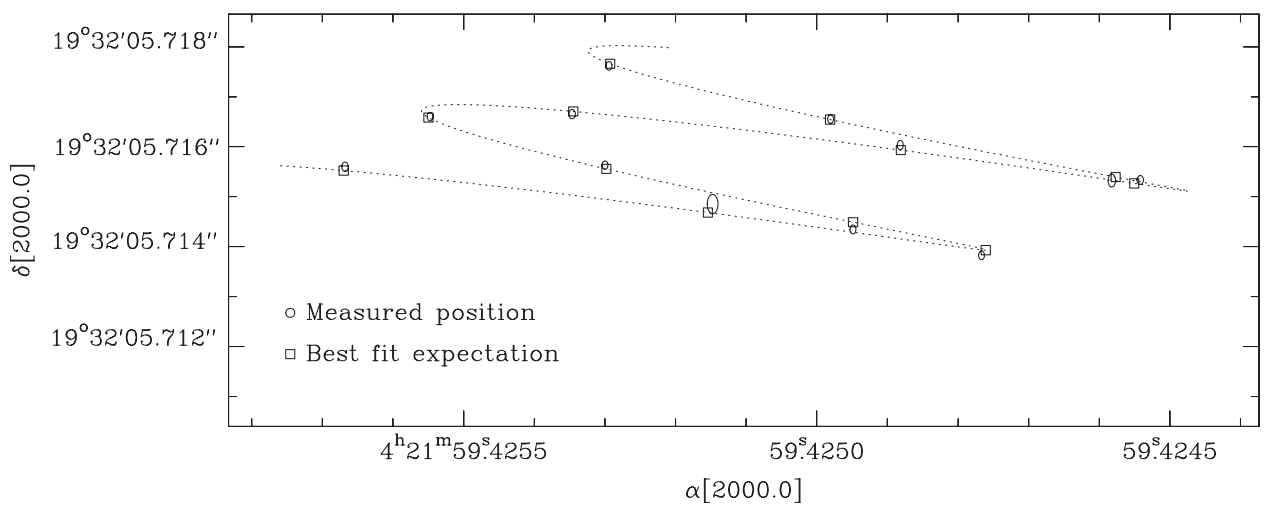

Figure 2. Trajectory of T Tau Sb on the plane of the sky as measured based on multi-epoch observations. The data were collected at 12 epochs from September 2003 to July 2005, and are shown as ellipses whose sizes represent the error bars. The dotted curve shows the best fit for a combination of parallax and proper motion. The squares are the positions predicted by the best fit at the 12 epochs (for details, see Loinard et al. 2007).

associated with IRAS $16293-2422$, they determine a distance of $178_{-37}^{+18}$ pc. Since the entire Ophiuchus complex is only approximately 10 pc across on the plane of the sky, it is very unlikely to be $60 \mathrm{pc}$ deep. Thus, if the distance determination of Imai et al. (2007) were confirmed, it would indicate that at least two unrelated star-forming regions coexist along the line of sight toward Ophiuchus. One region, associated with the core and north-eastern streamers, would be at $120 \mathrm{pc}$, while the region associated with the eastern cloud Lynds 1689 would be several tens of pc more distant. It would clearly be very important to obtain an independent confirmation of the distance to Lynds 1689.

\subsection{Taurus}

Taurus is perhaps the region that has been most instrumental to the development of our understanding of star formation (for a recent review, see Kenyon et al. 2008). Its mean distance has long been known to be about 140 pc (Kenyon et al. 1994), and the total extent of the region on the plane of the sky is approximately 10 degrees (or $25 \mathrm{pc}$ ). Roughly speaking, Taurus is composed of three parallel filaments, each $\approx 2$ degrees thick. Since those filaments have no reason to be orientated perpendicularly to the line of sight, it is quite conceivable that the near side of each filament might be roughly $25 \mathrm{pc}$ closer than its far side.

In a series of papers (Loinard et al. 2005, 2007; Torres et al. 2007, 2009, 2012), we have reported measurements of the trigonometric parallax of five young stars distributed across the Taurus complex. Three of these stars (Hubble 4, HDE 283572, and V773 Tau) are located toward the same portion of Taurus, associated with the prominent dark cloud Lynds 1495. Interestingly, all three stars are found to be at a similar distance $(132.8 \pm 0.5 \mathrm{pc}, 128.5 \pm 0.6 \mathrm{pc}$, and $132.9 \pm 2.4 \mathrm{pc})$. They also appear to share the same kinematics as measured by their proper motions and radial velocities, and therefore appear to belong to a coherent spatio-kinematic structure. The weighted mean of the three distance measurements $(131.4 \mathrm{pc})$ clearly provides a good estimate of the distance to that specific portion of Taurus. The dispersion about that mean $(2.4 \mathrm{pc})$, on the other hand, must provide of good estimate of the local depth of the Taurus complex. Interestingly, Lynds 1495 and its associated stellar population is of order 2 degrees across. This corresponds to a physical radius of $\sim 2.5 \mathrm{pc}$ for that region; the stellar population associated with Lynds 1495 appears to be roughly as deep as it is wide. 
The other two stars for which VLBI trigonometric parallaxes have been measured are $\mathrm{T}$ Tauri Sb and HP Tau/G3. They are located, respectively, to the southeast and northeast of the Taurus complex. T Tauri was found to be at $146.7 \pm 0.6 \mathrm{pc}$, while HP Tau is at $161.9 \pm 0.9 \mathrm{pc}$ (Loinard et al. 2007; Torres et al. 2009). Their proper motions are similar to one another, but significantly different from those of the stars associated with Lynds 1495. The errors in our distance measurements (a few pc) are approximately 10 times smaller than the separation $(\approx 30 \mathrm{pc})$ between the nearest and farthest stars in our sample. Thus, we are clearly resolving the depth of the Taurus complex along the line of sight.

Additional accurate distance measurements will be needed to reach definite conclusions regarding the three-dimensional structure of Taurus, but a few preliminary conclusions can be drawn. Taurus clearly appears to be roughly as deep as it is wide $(25-30 \mathrm{pc})$, with the near edge associated with the western side and the far edge corresponding to the eastern side. In that configuration, the filaments in Taurus would be oriented almost along the Galactic Center-Galactic anticenter axis (Ballesteros-Paredes et al. 2009). The space velocities of stars near the western edge of the complex are significantly different from those of the stars near the eastern edge.

Clearly, observations similar to those described here for several tens of young stars distributed across the entire complex would have the potential to reveal both the threedimensional structure and the internal kinematics of the stellar population associated with Taurus. This would have important consequences for our understanding of star formation.

\subsection{Perseus}

The Perseus cloud (Bally et al. 2008) contains a number of important subregions (for instance, NGC 1333 and IC 348; Walawender et al. 2008; Herbst 2008) where star formation is particularly active. For a long time, the distance to Perseus as a whole was assumed to be $350 \mathrm{pc}$, following Herbig \& Jones (1983). Specifically, this distance was for NGC 1333, and it was obtained from an analysis of several previous measurements. Somewhat later, Cernis et al. (1990) argued for a shorter distance to NGC 1333 (220 pc) based on extinction measurements. Many authors, however, have continued to use values between 300 and 350 pc. Indeed, it is not entirely clear if the different subregions are physically associated, and even if they are, there could be a significant distance spread because the entire region is of order 6 degrees across (corresponding to 25-30 pc).

Hirota et al. $(2008,2011)$ measured the distance to two young stellar objects in Perseus using VLBI observations of their associated water masers. They obtain a distance of $235 \pm 18$ pc to the source SVS 13 in NGC 1333 , and $232 \pm 18$ pc for a young stellar source associated with the dark cloud Lynds 1448 at the western edge of the Perseus complex. The projected separation between the two sources considered by Hirota et al. $(2008,2011)$ is approximately 1.5 degrees, or 6 pc. Their result that the two sources are at similar distances indicates that the western portion of Perseus can be assumed to be located at approximately 230-235 pc from the Sun. VLBI determination of the distance to young stars in the central part of Perseus (associated with B1) and of the eastern portions (B5 and IC 348) would enable a complete description of the structure of that important region.

\subsection{Serpens and Aquila}

The Serpens molecular cloud complex (Eiroa et al. 2008) is an important region of star formation, extending several degrees across the plane of the sky. Its distance has been a matter of some controversy over the years, with estimates ranging from $250 \mathrm{pc}$ to $650 \mathrm{pc}$ 
(for discussions, see Eiroa et al. 2008; Dzib et al. 2010). The distance used by most authors in the last 15 years is that reported by Straižys et al. (1996) based on stellar photometry: $d=259 \pm 37$ pc. More recently, Straižys et al. (2003) extended their work to include many more stars, covering an area of some 50 square degrees, and concluded that the front edge of the cloud was at $255 \pm 55 \mathrm{pc}$, and its depth approximately $80 \mathrm{pc}$. In projection, Serpens is located toward a larger complex of molecular clouds called the Aquila Rift (Dame \& Thaddeus 1985; Prato et al. 2008) believed to be at a distance of some 200 pc. The similarity between the distance to the Serpens cloud found by Straižys and coworkers, and that of the Aquila Rift suggests a physical association between the two.

Using VLBI observations, Dzib et al. (2010) measured the trigonometric parallax of the binary system EC95 in the Serpens core region. They obtain a distance of $414.9 \pm 4.4$ pc, significantly larger than the value usually adopted for Serpens. The Serpens core is a dense subregion of the Serpens cloud, with an extent of only approximately $5^{\prime}(0.6 \mathrm{pc})$ on the plane of the sky. As a consequence, the contribution of depth to the uncertainty of the distance to the Serpens core is expected to be very limited, and that distance can be assumed to be $415 \pm 5$ pc. The Serpens cloud, on the other hand is roughly 3 degrees across, or $25 \mathrm{pc}$ in diameter. The distance to the cloud as a whole should therefore be taken as $415 \pm 25 \mathrm{pc}$.

A detailed analysis of the possible reasons for the discrepancy between the most popular distance used for Serpens in the last 15 years, and that obtained directly from a trigonometric parallax is given in Dzib et al. (2010). Their conclusion is that the method used by Straižys et al. $(1996,2003)$ would naturally be sensitive to the first dust contribution along the line of sight, which might be associated with the clouds in the Aquila Rift rather than those of Serpens. In that interpretation, the Serpens and Aquila Rift clouds would not be physically associated, but merely located along the same line of sight. This is certainly not unexpected, since that line of sight is roughly in the direction of the Galactic plane. It will be very important to obtain VLBI parallax measurements of other stars in the direction of Serpens to confirm the distance measurement of Dzib et al. (2010) and clarify the relation between Serpens and the Aquila Rift.

\subsection{Orion}

Together with Taurus, Orion is undoubtedly the most often studied region of star formation. Efforts to measure the distance to Orion have largely been concentrated on the Orion Nebula region (an interesting and entertaining account of the history of the distance to Orion is given by Muench et al. 2008). For decades, the accepted distance was 480 pc, following Genzel et al. (1981). In the last five years, however, several VLBI parallax measurements have been published. Hirota et al. (2007) obtained a distance of $437 \pm 19$ pc using VLBI observations of water masers in the BN/KL region, whereas Sandstrom et al. (2007) found $389 \pm 23$ pc from VLBI continuum observations of a flaring star. The issue was settled by Menten et al. (2007) and Kim et al. (2008), who independently obtained highly consistent measurements ( $414 \pm 7 \mathrm{pc}$ and $418 \pm 6 \mathrm{pc}$, respectively).

Note, however, that the distances quoted so far are only for the Orion Nebula region. The Orion cloud complex is approximately 100 pc across, so different parts of the complex are most certainly at different distances. In addition, there are a number of dark clouds (e.g., Lynds 1617 and Lynds 1622; Reipurth et al. 2008) located in the vicinity of the Orion complex, but whose relation to the Orion clouds themselves is unclear. There are some indications that some of these clouds might be significantly closer than the Orion Nebula. Thus, a comprehensive VLBI program aimed at establishing the distance to the different parts of the Orion and surrounding clouds appears to be necessary. 


\section{The Gould's Belt Distances Survey}

The VLBI observations described so far have targeted a total of only of order a dozen different objects, but have already significantly refined our knowledge of the distance to the nearest regions of star formation. In several cases, they have provided the first direct indications of the three-dimensional structure of these regions.

One of the world's premier VLBI instruments (the Very Long Baseline Array; VLBA) has just undergone a major upgrade, which increased its bandwidth (and therefore its sensitivity) by a factor of several. Thanks to that upgrade, observations similar to those presented here will become feasible for several hundred young stars. Taking advantage of that possibility, we have initiated a large project, the 'Gould's Belt Distances Survey,' aimed at measuring the distance to some 200 young stars distributed across the five regions described in Section 2 (Ophiuchus, Taurus, Perseus, Serpens, and Orion). The first stage of the project (which has now been completed) used around 120 hours of observing time on the Expanded Very Large Array (EVLA) to identify adequate targets. Following that first stage, a total of some 2000 hours of VLBA time will be dedicated to the astrometric observations themselves. The VLBA observations have just started, and will last for a total of close to 4 years.

The final goal of the Gould's Belt Distances Survey is to estimate, with unprecedented accuracy, the mean distance, three-dimensional structure, and internal kinematics of the five regions described in Section 2. This will have important consequences both for the study of star formation, and for our understanding of the local structure of the Milky Way. In particular, our observations will shed new light on the origin of Gould's Belt. The results will be posted as they are obtained on the project's web site: http://www. crya. unam.mx/ 1. loinard/Gould/.

\section{Acknowledgements}

LL acknowledges support from the von Humboldt Stiftung, DGAPA, UNAM, and CONACyT, Mexico. The National Radio Astronomy Observatory is a facility of the National Science Foundation operated under cooperative agreement by Associated Universities, Inc.

\section{References}

André, P., Men'shchikov, A., Bontemps, S., et al. 2010, A\&A A, 518, L102

Ballesteros-Paredes, J., Gómez, G. C., Loinard, L., Torres, R. M., \& Pichardo, B. 2009, MNRAS, $395, \mathrm{~L} 81$

Bally, J. 2008, Handbook of Star Forming Regions, Vol. I, 459

Bally, J., Walawender, J., Johnstone, D., Kirk, H., \& Goodman, A. 2008, Handbook of Star Forming Regions, Vol. I, 308

Cernis, K. 1990, Ap\&SSS, 166, 315

Chini, R. 1981, A\&A, 99, 346

Dame, T. M. \& Thaddeus, P. 1985, ApJ, 297, 751

Dame, T. M., Hartmann, D., \& Thaddeus, P. 2001, ApJ, 547, 792

Dulk, G. A. 1985, ARA\&A, 23, 169

Dzib, S., Loinard, L., Mioduszewski, A. J., Boden, A. F., Rodríguez, L. F., \& Torres, R. M. $2010, A p J, 718,610$

Eiroa, C., Djupvik, A. A., \& Casali, M. M. 2008, Handbook of Star Forming Regions, Vol. II, 693

Evans, N. J., II, Dunham, M. M., Jørgensen, J. K., et al. 2009, ApJS, 181, 321

Genzel, R., Reid, M. J., Moran, J. M., \& Downes, D. 1981, ApJ, 244, 884

de Geus, E. J., de Zeeuw, P. T., \& Lub, J. 1989, A\& $A$, 216, 44 
Güdel, M., Arzner, K., Audard, M., et al. 2008, in: 14th Cambridge Workshop on Cool Stars, Stellar Systems, and the Sun, 384, 65

Herbig, G. H. \& Jones, B. F. 1983, AJ, 88, 1040

Herbst, W. 2008, Handbook of Star Forming Regions, Vol. I, 372

Hirota, T., Honma, M., Imai, H., Sunada, K., Ueno, Y., Kobayashi, H., \& Kawaguchi, N. 2010, $P A S J, 63,1$

Hirota, T., et al. 2008, PASJ, 60, 37

Hirota, T., et al. 2007, PASJ, 59, 897

Imai, H., et al. 2007, PASJ, 59, 1107

Kenyon, S. J., Gómez, M., \& Whitney, B. A. 2008, Handbook of Star Forming Regions, Vol. I, 405

Kenyon, S. J., Dobrzycka, D., \& Hartmann, L. 1994, AJ, 108, 1872

Kim, M. K., et al. 2008, PASJ, 60, 991

Knude, J. \& Hog, E. 1998, A\&A, 338, 897

Lockman, F. J., Benjamin, R. A., Heroux, A. J., \& Langston, G. I. 2008, ApJ, 679, L21

Loinard, L., Torres, R. M., Mioduszewski, A. J., Rodríguez, L. F., González-Lópezlira, R. A., Lachaume, R., Vázquez, V., \& González, E. 2007, ApJ, 671, 546

Loinard, L., Mioduszewski, A. J., Rodríguez, L. F., González, R. A., Rodríguez, M. I., \& Torres, R. M. 2005, ApJ, 619, L179

Loinard, L., Torres, R. M., Mioduszewski, A. J., \& Rodríguez, L. F. 2008, ApJ, 675, L29

Lombardi, M., Lada, C. J., \& Alves, J. 2008, A\& A, 480, 785

Menten, K. M., Reid, M. J., Forbrich, J., \& Brunthaler, A. 2007, A\& A, 474, 515

Muench, A., Getman, K., Hillenbrand, L., \& Preibisch, T. 2008, Handbook of Star Forming Regions, Vol. I, 483

Perryman, M. A. C., et al. 1997, A\&A, 323, L49

Poppel, W. 1997, Fund. Cosmic Phys., 18, 1

Prato, L., Rice, E. L., \& Dame, T. M. 2008, Handbook of Star Forming Regions, Vol. I, 18

Reipurth, B., Megeath, S. T., Bally, J., \& Walawender, J. 2008, Handbook of Star Forming Regions, Vol. I, 782

Sandstrom, K. M., Peek, J. E. G., Bower, G. C., Bolatto, A. D., \& Plambeck, R. L. 2007, ApJ, 667,1161

Schaefer, G. H., Simon, M., Prato, L., \& Barman, T. 2008, AJ, 135, 1659

Straižys, V., \& Černis, K., Bartašiūtè, S. 2003, A\&A A, 405, 585

Straižys, V., Černis, K., \& Bartašiūte, S. 1996, Balt. Astron., 5, 125

Thompson, A. R., Moran, J. M., \& Swenson, G. W., Jr. 2001, Interferometry and synthesis in radio astronomy, $2^{\text {nd }}$ ed., New York: Wiley

Torres, R. M., Loinard, L., Mioduszewski, A. J., \& Rodríguez, L. F. 2009, ApJ, 698, 242

Torres, R. M., Loinard, L., Mioduszewski, A. J., \& Rodríguez, L. F. 2007, ApJ, 671, 1813

Torres, R. M., Loinard, L., Mioduszewski, A. J., et al. 2012, ApJ, 747, 18

Walawender, J., Bally, J., Francesco, J. D., Jørgensen, J., \& Getman, K. 2008, Handbook of Star Forming Regions, Vol. I, 346

Ward-Thompson, D., Di Francesco, J., Hatchell, J., et al. 2007, PASP, 119, 855

Wilking, B. A., Gagné, M., \& Allen, L. E. 2008, Handbook of Star Forming Regions, Vol. II, 351 